Revista de Matemática: Teoría y Aplicaciones 1994 1(1) : 1-16

CIMPA - UCR - CCSS ISSN: 1409-2433

\title{
EQUILIBRIO WALRASIANO EN ECONOMÍAS DE INTERCAMBIO
}

\author{
Osvaldo Acuña Ortega* Fernán Ulate Montero**
}

\begin{abstract}
Resumen
Se presentan las funciones de exceso de demanda agregada de una economía de intercambio. Se estudian las propiedades y los vectores de precios de equilibrio de dichas funciones. Se prueba que el equilibrio walrasiano es un óptimo de Pareto. Se estudia el concepto de economía regular y se prueba la existencia de equilibrio walrasiano, para dicha economía. Por último, estudiamos la unicidad del vector de precios de equilibrio.
\end{abstract}

\begin{abstract}
We present the aggregated excess demand functions in an exchange economy. We study the properties and the equilibrium price vectors of the aggregated excess demand functions. We prove that the walrasian equilibrium is a Pareto optimun. We study regular ecomies. We prove the existence of regular equilibrium for regular economies. We study the unicity of the equilibrium price vector.
\end{abstract}

\section{Introducción}

Los orígenes de la teoría del equilibrio general pueden remontarse a A. Smith. Posteriormente a Smith, ya algunos autores como A. Cournot y H. Mangoldt, trabajaron en la teoría del equilibrio pero sin introducir los aspectos subjetivos que otros economistas como C. Menger, S. Jevons y L. Walras introdujeron a través del concepto de utilidad marginal, el cual a su vez tiene sus orígenes en H. Gossen y A. Dupuit.

Walras extiende la teoría de cambio al caso de tres o más bienes. La exposición matemática de Walras ha tenido un impacto muy grande en teoría económica que aún hoy, a pesar de los trabajos posteriores, tiene un valor imponderable. Walras estudió varias propiedades del modelo de equilibrio general, como la estabilidad (cuyo proceso dinámico para alcanzar el equilibrio posee una belleza incuestionable), la optimalidad (que fue estudiada más a

\footnotetext{
*Escuela de Matemática, Universidad de Costa Rica, 2060 San José, Costa Rica.

** Escuale de Economía, Universidad de Costa Rica, 2060 San José, Costa Rica.
} 
fondo por sus discípulos, por ejemplo W. Pareto), la existencia cuyo estudio ha sido bastante superado en el siglo XX, también merece reconocimiento, etc. W. Pareto, E. Barone, K. Wicksell, J. B. Clark, son algunos autores que posteriormente hicieron contribuciones importantes a esta teoría del equilibrio general. Después, se estudió el tema mediante el uso de topología general y teoría de conjuntos. Esta metodología permitió conocer más a fondo el problema de la existencia. Podemos citar, entre muchos otros, a J. Von Neumann, K. Menger, A. Wald, L. W. McKenzie, D. Gale, H. Nikaido, K. Arrow, G. Debreu. En los últimos años la topología diferencial ha permitido llevar la teoría del equilibrio por un rumbo muy fructífero. Dentro de esta metodología, el concepto de economía regular (que se remonta a G. Debreu, 1970), ocupa un lugar central. Una vez definido el concepto de economía regular, gran cantidad de autores encuentran en él el instrumento ideal para el desarrollo riguroso de la teoría del equilibrio walrasiano. Podemos citar entre otros a E. Dierker, H. Dierker, Y. Balasko, S. Smale, T. Kehoe, A. Mas. Colell.

El trabajo pionero de L. Walras, a pesar de la gran cantidad de esfuerzos que se han hecho posteriormente, sigue teniendo todavía una importancia primordial. Su análisis dinámico para llegar al punto de equilibrio, sus definiciones de los diferentes tipos de ecuaciones matemáticas que describen una economía, su creencia en la importancia que debe dársele a la inclusión de todos los sectores en el análisis económico, y su extraordinario supuesto de que las transacciones se realizan en el punto de equilibrio. Su fé en las matemáticas como vehículo para comprender y transmitir la compresión de la realidad económico-social, su creencia firme en la necesidad que tiene el conocimiento científico de modelar los fénomenos, su metodología de ir de lo extraordinariamente simple a lo complejo, su integración de temas subjetivos y objetivos, son aspectos de su pensamiento que tienen gran importancia en nuestra época. Leon Walras fue un hombre que se adelantó mucho a su tiempo, su "Equilibrio Económico", como dice el monumento que le dedicó la Universidad de Lausana, lo ubicó para siempre, entre los más grandes economistas de todos los tiempos.

En este trabajo estudiaremos una economía con varios bienes y varios individuos sin producción, tal como lo hizo Walras en su "Segunda Memoria". A diferencia de Walras y siguiendo más bien a Pareto, suponemos que cada individuo tiene una relación de prefencias y no una función de utilidad. Dada la relación de preferencias y una canasta inicial de bienes, los individuos, maximizando su bienestar, obtienen una función de exceso de demanda para un nivel cualquiera de precios. Estas funciones individuales, se suman para todos los consumidores y se obtiene un vector de funciones de exceso de demanda agragada, que llamamos $f(p)$. Como consecuencia de los supuestos del modelo, $f(p)$ tiene una serie de propiedades muy interesantes, como por ejemplo: es continua y diferenciable, es homogénea de grado cero en precios, cumple con la ley de Walras, esto es $p \cdot f(p)=0$, proposiciones 2 y 6 .

Ahora bien, Walras suponía que si $f(p) \neq 0$, no se realizaban transacciones. A través de su famoso proceso de tâtonnement, los precios de cada bien subían (bajaban) si el exceso de demanda de ese bien era positivo (negativo) hasta llegar al punto $f(p)=0$, que llamamos equilibrio walrasiano de precios y al $p$ correspondiente, vector de precios de equilibrio. El equilibrio walrasiano de precios resulta ser un equilibrio de precios (proposición 3), y por lo tanto, bajo los supuestos dados, un óptimo de Pareto (proposición 4). El resultado anterior es muy importante, desde el punto de vista científico y de política económica. El equilibrio 
walrasiano de precios es, al mismo tiempo, un marco de referencia teórico de mucha utilidad y un punto deseable, que la política debería buscar, por ser un óptimo. Un problema muy importante es entonces, la existencia del equilibrio walrasiano de precios. L. Walras se enfrentó al problema contando ecuaciones e incógnitas, pero a pesar de que para una época, tal metodología era un aporte a la teoría económica, el procedimiento estaba esencialmente equivocado; en efecto, la igualdad entre el número de ecuaciones y de incognitas no es necesaria ni suficiente para obtener un resultado económicamente significativo (la solución no puede tener númerosos negativos o imaginarios).

El concepto de economía regular brinda una manera práctica y novedosa de estudiar el problema de existencia del equilibrio. La definición 5 nos dice que un vector de precios de equilibrio es regular si el rango de $\partial f(p)$ es $l-1$, donde $l$ es el número de bienes en la economía, y la definición 6 nos dice que una economía es regular, si todos sus vectores de precios de equilibrio son regulares. En 1972, E. Dierker probó que la función de exceso de demanda tiene un número impar de ceros en una economía regular. Su resultado es una consecuencia directa del Teorema del Indice. En este artículo se dan todos los detalles técnicos requeridos, para satisfacer las hipótesis del Teorema de Poincaré-Hopf (Teorema B del apéndice) y así obtener el Teorema del Indice como consecuencia de este resultado clásico.

Definimos el índice de un equilibrio de precios regular (definición 7) como:

$$
\text { índice }(p)=\operatorname{signo}\left(\operatorname{det}\left[\begin{array}{cc}
-\partial f(p) & p \\
-p^{t} & 0
\end{array}\right]\right) .
$$

El Teorema del Indice (teorema 17), es el resultado de más importancia de todo el trabajo nos dice que si, E es el conjunto de precios de equilibrio de una economía regular $\mathcal{E}$, entonces $\sum_{p \in \mathrm{E}}$ índice $(p)=1$. Este teorema que tiene como corolario (corolario 18) el hecho que $\mathrm{E}$ tiene un número impar de elementos. En particular $\mathrm{E} \neq \emptyset$, lo que implica desde luego, la existencia del equilibrio $p$ con todas sus entradas reales y positivas, por lo tanto con significado económico. Lo anterior, puesto que el $p$, que hemos demostrado que existe, está en $S_{++}^{l-1}=\left\{p \in \mathbb{R}_{++}^{l} /\|p\|=1\right\}$.

El corolario 19 nos da condiciones necesarias y suficientes para la unicidad del equilibrio, tema que también ha tenido mucha importancia en la literatura económica.

Si bien la presentación y pruebas de este artículo, tienes sus propias particularidades, nuestro trabajo está inspirado en los trabajos de G. Debreu (1970), E. Dierker (1972), A. Mas. Colell (1985) y en nuestros artículos anteriores, Acuña, O. y Ulate, F. (1991, 1992).

A continuación presentamos una serie de definiciones, que ya hemos dado en trabajos anteriores (1991, 1992).

Notación Sea $p \in \mathbb{R}^{l}, p \geqq 0, p \geq 0, p>0$ denotan las siguientes condiciones respectivamente $p_{i} \geq 0$ para todo $i, p \geqq 0$ y $p \neq 0, p_{i}>0$ para todo $i$. Para $p, p^{\prime} \in \mathbb{R}^{l}$, decimos que $p \geqq p^{\prime}, p \geq p^{\prime}, p>p^{\prime}$ si se satisface respectivamente, $p-p^{\prime} \geqq 0, p-p^{\prime} \geq 0, p-p^{\prime}>0$.

i) $\mathbb{R}_{++}^{l}$ denota el conjunto de todos los vectores $x \in \mathbb{R}^{l}$ y $x>0$.

ii) Sea $\succeq$ una relación de preferencias en $\mathbb{R}_{++}^{l}$ 
(a) $\succeq$ se dice ser estrictamente monotónica, si $v \geq x$ implica $v \succ x(v \succ x$ denota la condición $v \succeq x$ y $\neg(x \succeq v))$

(b) $\succeq$ es continua si $\succeq \subseteq \mathbb{R}_{++}^{l} \times \mathbb{R}_{++}^{l}$ es cerrado

(c) $\succeq$ es convexa si $x \succeq z$ implica $\alpha x+(1-\alpha) z \succeq z$, para todo $0 \leq \alpha \leq 1$

(d) $\succeq$ es estrictamente convexa si $x \succeq z$ y $x \neq z$ implica $\alpha x+(1-\alpha) z \succ z$

(e) $\succeq$ satisface la condición de frontera si para todo $x \in \mathbb{R}_{++}^{l}$ el conjunto $\left\{x \in \mathbb{R}_{++}^{l} / z \succeq x\right\}$ es cerrado $\mathbb{R}^{l}$

(f) $\succeq$ es de clase $C^{r}$ (o simplemente $C^{r}$ ), $r \geq 0$ si $\partial \succeq=\left\{(x, z) \in \mathbb{R}_{++}^{l} \times \mathbb{R}_{++}^{l} / x \sim z\right\}$ es una $C^{r}$-subvariedad de $\mathbb{R}_{++}^{l} \times \mathbb{R}_{++}^{l}$ de dimensión $2 l-1$ ( $x \sim z$ denota la condición $x \succeq z$ y $z \succeq x)$ y además para todo $x \in \mathbb{R}_{++}^{l}$ y todo $\epsilon \geq 0$ existe $z \in \mathbb{R}_{++}^{l}$ tal que $z \succ x$ y $\|z-x\|<\delta$ ( $\succeq$ es localmente no sociada)

(g) $\succeq$ es I.E.C. si es $\mathrm{C}^{r}, r \geq 2$ en $\mathbb{R}_{++}^{l}$, para cada $x \in \mathbb{R}_{++}^{l}$ la curvatura gaussiana $\mathrm{C}_{x}$ de $x$ en $I_{x}=\left\{y \in \mathbb{R}_{++}^{l} / x \sim y\right\}$ variedad de indeferencia de $x$ (Ver AcuñaUlate, 1991) es diferente de 0 y además la relación de preferencia es estrictamente convexa

iii) (a) Una asignación es un vector $x=\left(x_{i}\right)$ en $\left(\mathbb{R}_{++}^{l}\right)^{n}$

(b) Una asignación $x=\left(x_{i}\right)$ es alcanzable si $\sum_{i=1}^{n} x_{i} \leq \sum_{i=1}^{n} w_{i}$, donde $w_{i}$ es la canasta inicial de bienes del individuo $i$.

iv) Una asignación alcanzable $x$ es un equilibrio de precios si existe un vector $p \geq 0$, $p \in \mathbb{R}^{l}$, tal que $p \cdot \sum_{i=1}^{n} w_{i} \leq p \cdot \sum_{i=1}^{n} x_{i}$ y para todo $i$, si $x_{i}^{\prime} \succ_{i} x_{i}$, entonces $p x_{i}^{\prime}>p x_{i}$.

v) Una asignación alcanzable $x$ es óptima si no existe ninguna otra asignación alcanzable $x^{\prime}$ tal que, para cada $i, x_{i}^{\prime} \succeq_{i} x_{i}$ y existe $j$ con $x_{j}^{\prime} \succ x_{j}$.

\section{Desarrollo y pruebas matemáticas}

En este trabajo supondremos una situación de intercambio, sin producción. Nuestro modelo de la economía consta de $l$ bienes y $n$ individuos, donde $n$ es un número finito pero muy grande, de tal manera que las acciones de un individuo no afectan el precio de los bienes.

Hay perfecta información; completa libertad de entrada, salida, contratación y consumo; no hace diferencia con quien se contrate ni hay externalidades. Los consumidores son racionales en el sentido de que maximizan un nivel de bienestar de acuerdo a su orden de preferencias.

Con los supuestos anteriores en mente, definiremos una economía de intercambio.

Definición 1 Una economía de intercambio $\mathcal{E}$ es una colección de $n$ individuos, cada uno de los cuales posee una relación de preferencias $\succeq_{i}$ definida en $\mathbb{R}_{++}^{l}$ y una canasta inicial de bienes $w_{i} \in \mathbb{R}_{++}^{l}$.

Suponiendo que las preferencias de todos los individuos son estrictamente convexas, continuas y estrictamente monotónicas, la maximización de bienestar por parte de cada individuo, nos permite obtener una función de demanda $g_{i}: \mathbb{R}_{++}^{l} \longrightarrow \mathbb{R}_{++}^{l}$, para cada $i$, 
tal que $g_{i}(q)=\varphi_{i}\left(\succeq_{i}, q, q \cdot w_{i}\right)$, donde $\varphi_{i}$ es la función de demanda de la proposición 3 de Acuña-Ulate (1991).

La función $f_{i}: \mathbb{R}_{++}^{l} \longrightarrow \mathbb{R}^{l}$ definida por $f_{i}(p)=g_{i}(p)-w_{i}$ es la función de exceso de demanda del individuo $i$.

Definición 2 Llamaremos $f: \mathbb{R}_{++}^{l} \longrightarrow \mathbb{R}^{l}$ dada por $f(p)=\sum_{i=i}^{n} f_{i}(p)$, la función de exceso de demanda agregada.

Proposición 1 Si $\succeq_{i}$ es un orden de preferencias en $\mathbb{R}_{++}^{l}$ tal que $\succeq_{i}$ es estrictamente monotónico, continuo, estrictamente convexo y satisface la condición de frontera. Entonces para toda secuencia $\left(p_{m}\right)$ en $\mathbb{R}_{++}^{l}$ tal que $p_{m} \longrightarrow p, p \neq 0, p$ en la frontera de $\mathbb{R}_{++}^{l}$ se tiene que $\left\|f_{i}\left(p_{m}\right)\right\| \longrightarrow+\infty$, donde $f_{i}$ es la función de exceso de demanda de $\succeq_{i}$ y $w_{i} \in \mathbb{R}_{++}^{l}$.

Prueba Como $f_{i}(p)=\varphi_{i}\left(p, w_{i} \cdot p\right)-w_{i}$, con $\varphi_{i}$ la función de demanda, es suficiente probar que la secuencia $y_{m}=\varphi_{i}\left(p_{m}, w_{i} \cdot p_{m}\right)$ es tal que $\left\|y_{m}\right\| \longrightarrow+\infty$, dado que $\left\|y_{m}\right\| \leq$ $\left\|f_{i}\left(p_{m}\right)\right\|+\left\|w_{i}\right\|$.

Probaremos primero que no existe elemento en $B\left(p, p \cdot w_{i}\right)$ que maximice $\succeq_{i}$ en $B\left(p, p \cdot w_{i}\right)$. Sea $x \in B\left(p, p \cdot w_{i}\right)$, como $p$ pertenece a la frontera de $\mathbb{R}_{++}^{l}$ existe $j$ tal que $p_{j}=0$, por lo tanto $x+e_{j} \succ x$ y $\left(x+e_{j}\right) \cdot p=p \cdot x \leq w_{i}$, luego $x+e_{j} \in B\left(p, p \cdot w_{i}\right)$.

Suponga por contradicción que $\left\|y_{m}\right\|$ no converge a $\infty$, esto implica que existe una subsecuencia $x_{k}=y_{m_{k}}$ acotada. Podemos suponer que $x_{k} \longrightarrow z$, para algún $z$.

Observemos primero que $z \in \mathbb{R}_{++}^{l}$. Suponga que $z \notin \mathbb{R}_{++}^{l}$, como $x_{k} \longrightarrow z$, entonces $z$ pertenece a la frontera de $\mathbb{R}_{++}^{l}$, luego existe $j_{0}$ tal que $z_{j_{0}}=0$.

Sean $s=\operatorname{máx}_{p_{j} \neq 0}\left(w_{i} / p_{j}\right)+1, v(0)=\sum_{j \neq j_{0}} s e_{j}$ y $x \in \mathbb{R}_{++}^{l}$ tal que $p \cdot x<w_{i} \cdot p$. Como $\succeq$ satisface la condición de frontera, entonces $L_{x}=\left\{y \in \mathbb{R}_{++}^{l} / y \succeq_{i} x\right\}$ es cerrado en $\mathbb{R}^{l}$, luego el complemento de $L_{x}$ en $\mathbb{R}^{l}$ es abierto en $\mathbb{R}^{l}$, por lo tanto existe $\sigma>0$ tal que $\left\{y \in \mathbb{R}^{l} /\left\|y-v_{0}\right\| \leq \sigma\right\}$ está contenido en el complemento de $L_{x}$ en $\mathbb{R}^{l}$ y entonces para cada $x^{\prime} \in \mathbb{R}_{++}^{l}$ con $\left\|v(0)-x^{\prime}\right\| \leq \sigma$ tenemos $x \succ_{j} x^{\prime}$. En particular para $v(\sigma)=v(0)+\sigma e_{j_{0}}$ se tiene $x \succ_{i} v(\sigma)$.

Por definición de $s$ tenemos que $z_{j}<s$, para todo $j$. Como $x_{k} \longrightarrow z$, existe $k_{0}$ tal que si $k \geq k_{0}$ entonces $x_{k}^{j}<s$ para $j \neq j_{0}$ y $x_{k}^{j}<\sigma$ para $j=j_{0}$, luego $v(\sigma)>x_{k}$ y entonces $x \succ_{i} x_{k}$ para todo $k \geq k_{0}$. Dado que $p_{m_{k}} \longrightarrow p$ existe $k_{1}$, tal que para $k \geq k_{1} p_{m_{k}} \cdot x<p_{m_{k}} \cdot w_{i}$, esto implica que $x_{k} \succeq_{i} x$. Para $k$ suficientemente grande $\left(k>\operatorname{máx}\left\{k_{0}, k_{1}\right\}\right)$ tenemos que $x \succ_{i} x_{k}$ y $x_{k} \succeq_{i} x$, lo cual es una contradicción, luego $z \in \mathbb{R}_{++}^{l}$.

Por otro lado existe $y \in B\left(p, w_{i} \cdot p\right)$ tal que $y \succ_{i} z$, entonces por continuidad de $\succeq_{i}$ para $\sigma>0$ muy pequeño y $j_{1}$, tal que $p_{j_{1}} \neq 0$ tenemos que $y-\sigma e_{j_{1}} \succeq_{i} z$. Puesto que $x_{k} \longrightarrow z$ existe $k_{0}$ tal que si $k \geq k_{0}, y-\sigma e_{j_{1}} \succeq_{i} x_{k}$. Como $p\left(y-\sigma e_{j_{1}}\right) \leq w_{i} \cdot p-\sigma p_{j_{1}}<w_{i} \cdot p$ y $p_{m_{k}} \longrightarrow p$, existe $k_{1}$ tal que $k \geq k_{1}$, implica $p_{m_{k}}\left(y-\sigma e_{j_{1}}\right)<w \cdot p_{m_{k}}$, entonces $x_{k} \succ_{i} y-\sigma e_{j_{1}}$, $\forall k \geq k_{1}$. Para $k$ suficientemente grande se tendría que $x_{k} \succ_{i}\left(y-\sigma e_{j_{1}}\right)$ y $y-\sigma e_{j_{1}} \succ_{i} z$ lo cual es una contradicción. Por lo tanto $z$ sólo puede ser máximo para $\succeq_{i}$ en $B\left(p, p \cdot w_{i}\right)$, pero tales elementos no pueden existir, luego $\left\|y_{m}\right\| \longrightarrow+\infty$.

Proposición 2 Dada una economía $\mathcal{E}$ de intercambio con órdenes de preferencia estrictamente monotónicos, continuos y estrictamente convexos, entonces la función de exceso de demanda agregada $f: \mathbb{R}_{++}^{l} \longrightarrow \mathbb{R}^{l}$, satisface las siguientes propiedades:

(i) $f$ es continua. 
(ii) $p \cdot f(p)=0$ para cada $p$ en $\mathbb{R}_{++}^{l}$, conocida como ley de Walras.

(iii) $f(\alpha p)=f(p)$ para cada $p$ y $\alpha \in \mathbb{R}, \alpha>0$; las funciones exceso de demanda son homogéneas de grado 0.

Si las preferencias satisfacen la condición de frontera se tiene también:

(iv) Si $p_{h} \longrightarrow p, p_{h} \in \mathbb{R}_{++}^{l}, p$ en la frontera de $\mathbb{R}_{++}^{l}$ y $p \neq 0$, entonces $\left\|f\left(p_{h}\right)\right\| \longrightarrow+\infty$.

\section{Prueba}

(i) $f(p)=\sum_{i=1}^{n} f_{i}(p)=\sum_{i=1}^{n} g_{i}(p)-\sum_{i=1}^{n} w_{i}$, como $g_{i}$ es continua (proposición 5 , AcuñaUlate (1991)) para cada $i$, se tiene que $f$ es continua.

(ii) Sabemos que $g_{i}(p) \cdot p=w_{i} \cdot p$ para cada $i=1, \ldots, n$ (proposición 3, Acuña-Ulate (1991)) y sumando sobre $i$ obtenemos $\left(\sum_{i=1}^{n}\left(g_{i}(p)-w_{i}\right)\right) \cdot p=0$, es decir $f(p) \cdot p=0$.

(iii) $f(\alpha p)=\sum_{i=1}^{n} g_{i}(\alpha p)-\sum_{i=1}^{n} w_{i}$, pero $g_{i}(\alpha p)=g_{i}(p)$ para cada $i$, por lo que $f(\alpha p)=$ $f(p)$.

La proposición 4 nos da (iv).

Definición 3 Llamamos vector de precios de equilibrio, al vector $p \in \mathbb{R}^{l}, p \geq 0$, tal que $f(p)=0$.

Definición 4 Una asignación alcanzable $x=\left(x_{i}\right)$ es un equilibrio walrasiano (de precios) si existe un vector $p \in \mathbb{R}^{l}, p \geq 0$, tal que, para cada $i, x_{i}$ maximiza $\succeq_{i}$ en el conjunto $B\left(p, p \cdot w_{i}\right)=\left\{z \in \mathbb{R}_{++}^{l} / p z \leq p \cdot w_{i}\right\}$.

Nota Si las preferencias $\succeq_{i}$ son estrictamente monotónicas, entonces para un equilibrio walrasiano $\left(x_{i}\right)$, el vector $p$ correspondiente es tal que $p>0$. Si existe $p^{j}=0$, no existiría maximizador en $B\left(p, p \cdot w_{i}\right)$ para $\succeq_{i}$, ya que a cualquier canasta en $B\left(p, p \cdot w_{i}\right)$ se le puede aumentar la cantidad del bien $j$ y siempre va a estar en $B\left(p, p \cdot w_{i}\right)$.

Proposición 3 Sean las preferencias estrictamente monotónicas, continuas y convexas. Si $x=\left(x_{i}\right)$ es un equilibrio walrasiano, entonces $\sum_{i=1}^{n} x_{i}=\sum_{i=1}^{n} w_{i}$.

Prueba Sea $p$ el vector de equilibrio de precios, como $p>0, \sum_{i=1}^{n}\left(w_{i}-x_{i}\right) \geq 0,\left(\left(x_{i}\right)\right.$ es alcanzable) y $\sum_{i=1}^{n}\left(w_{i}-x_{i}\right) \cdot p=0$, puesto que como $x_{i}$ es maximal en $B\left(p, p \cdot w_{i}\right)$ se tiene que $x_{i} \cdot p=w_{i} \cdot p$ dado que $\succeq_{i}$ es estrictamente monotónica y sumando sobre la ecuación anterior obtener la ecuación deseada. Así se debe tener $\sum_{i=1}^{n}\left(w_{i}-x_{i}\right)=0$.

Proposición 4 Si las preferencias son estrictamente monotónicas, continuas y estrictamente convexas, para cada $p \in \mathbb{R}^{l}, p>0$ las siguientes afirmaciones son equivalentes:

(i) $p$ es un vector de precios correspondientes a algún equilibrio walrasiano $\left(x_{i}\right)$

(ii) $x_{i}=f_{i}(p)+w_{i}, i=1, \ldots, n$ es una asignación alcanzable

(iii) $x_{i}^{\prime}=x_{i}$ para todo $i=1, \ldots, n$, donde $x_{i}^{\prime}$ maximiza $\succeq_{i}$ en $B\left(p, p \cdot w_{i}\right)$

(iv) $f(p)=0$. 


\section{Prueba}

$(\mathrm{i}) \Longrightarrow$ (iii) Como $x_{i}^{\prime}$ maximiza $\succeq_{i}$ en $B\left(p, p \cdot w_{i}\right), x_{i}^{\prime}=g_{i}(p)=f_{i}(p)+w_{i}=x_{i}$ por la unicidad de la función demanda.

(iii) $\Longrightarrow$ (ii) Es trivial.

(ii) $\Longrightarrow$ (i) $x_{i}=f_{i}(p)+w_{i}=g_{i}(p)$ define una asignación alcanzable que cumple la condición de ser equilibrio walrasiano con $p$ vector de precios correspondiente.

(i) $\Longrightarrow$ (iv) Por el corolario 3 tenemos que $\sum_{i=1}^{n} x_{i}^{\prime}=\sum_{i=1}^{n} w_{i}$ y como $x_{i}^{\prime}-w_{i}=f_{i}(p)$ para cada $i=1, \ldots, n$ tenemos $\sum_{i=1}^{n} f_{i}(p)=0$ es decir $f(p)=0$.

(iv) $\Longrightarrow$ (ii) Si $f(p)=0$, entonces $\sum_{i=1}^{n}\left(f_{i}(p)+w_{i}\right)=\sum_{i=1}^{n} w_{i}$. En particular se tiene que $\sum_{i=1}^{n}\left(f_{i}(p)+w_{i}\right) \leq \sum_{i=1}^{n} w_{i}$, por lo tanto $x_{i}=f_{i}(p)+w_{i}$ define una asignación alcanzable.

Nota Por el teorema anterior, es claro que todo vector de precios correspondiente a un equilibrio walrasiano (definición 4) es un vector de equilibrio de precios (definición 3) y viceversa.

Proposición 5 Si las pertenencias son estrictamente monotónicas, continuas y convexas, entonces toda asignación $x=\left(x_{i}\right)$ que es un equilibrio walrasiano de precios es un óptimo. Prueba Por la proposición 2.5 de Acuña-Ulate (1992), es suficiente probar que $\left(x_{i}\right)$ es un equilibrio de precios compensado. Sea $p>0$ vector de equilibrio de precios para $\left(x_{i}\right)$, por la proposición 3 tenemos $\sum_{i=1}^{n} p \cdot x_{i}=\sum_{i=1}^{n} p \cdot w_{i}$. Por otro lado sea $x_{i}^{\prime}$ una canasta tal que $p \cdot x_{i}^{\prime}<p \cdot x_{i}$, como $p \cdot x_{i}^{\prime} \leq p \cdot w_{i}$, tenemos que $x_{i}^{\prime} \in B\left(p, w \cdot p_{i}\right)$ y entonces $x_{i} \succeq_{i} x_{i}^{\prime}$, como $x_{i}^{\prime} \cdot p<p \cdot w_{i}$ se debe tener $x_{i} \succ x_{i}^{\prime}$. Hemos probado que $x_{i}^{\prime} \succeq x_{i}$ implica $p \cdot x_{i} \leq p \cdot x_{i}^{\prime}$. Luego $\left(x_{i}\right)$ es un equilibrio de precios compensado.

De aquí en adelante supondremos que todos los individuos de la economía $\mathcal{E}$ tienen sus preferencias $\mathrm{C}^{r}, r \geq 2$ y satisfacen la condición de frontera.

Proposición 6 Si las preferencias son estrictamente monotónicas, continuas y I.E.C., entonces la función $f: \mathbb{R}_{++}^{l} \longrightarrow R^{l}$ de exceso de demanda agregada, es $\mathrm{C}^{r-1}$.

Prueba Como $f=\sum_{i=1}^{n} f_{i}$ y $f_{i}$ es $\mathrm{C}^{r-1}$, por proposición 15 de Acuña-Ulate (1991), $f$ es $\mathrm{C}^{r-1}$.

Definición 5 Suponiendo las hipótesis de la proposición 6, decimos que un vector de precios de equilibrio $p$ es regular si el rango de $\partial f(p)$ es $l-1$.

Definición 6 Una economía $\mathcal{E}$ es regular si todo vector de precios de equilibrio en $\mathcal{E}$ es regular.

Lema 7 Sea $A$ una matriz $l \times l$ tal que para $p \in \mathbb{R}^{l}$ se tiene $A p=0$ y $A\left(T_{p}\right) \subseteq T_{p}$, donde $T_{p}=\left\{q \in \mathbb{R}^{l} / p q=0\right\}$, entonces $\operatorname{det}\left(A / T_{p}\right)=\frac{1}{\|p\|^{2}} \operatorname{det}\left[\begin{array}{cc}A & p \\ -p^{t} & 0\end{array}\right]$, donde $A / T_{p}$ es la transformación lineal $T_{p} \longrightarrow T_{p}$ tal que $\left(A / T_{p}\right)(v)=A v$.

Prueba Sea $\left(p_{1}, \ldots, p_{l-1}\right)$ base ordenada de $T_{p}$, entonces $\left(p_{1}, \ldots, p_{l-1}, p\right)$ es también base ordenada de $\mathbb{R}^{l}$. Denote por $H=\left(h_{i j}\right)$ la matriz $(l-1) \times(l-1)$ que representa a $A / T_{p}$ en la base $\left(p_{1}, \ldots, p_{l-1}\right)$, se tiene que $A p_{i}=\sum_{k=1}^{n-1} h_{k i} p_{k}$, para $i=1, \ldots, l-1$.

Sea $P=\left(p_{1}, \ldots, p_{l-1}, p /\|p\|\right)$ matriz $l \times l$ definida por columnas. $P$ es invertible $\mathrm{y}$ si $\left(e_{1}, \ldots, e_{l}\right)$ es la base canónica de $\mathbb{R}^{l}$, entonces $A P e_{i}=A p_{i}=\sum_{k=1}^{n-1} h_{k i} p_{k}$, para $i=$ $1, \ldots, l-1$ y $A P e_{n}=A \cdot(p /\|p\|)=0$. Tenemos también que 


$$
P \cdot\left[\begin{array}{cc}
H & 0 \\
0 & 0
\end{array}\right] e_{i}=P \cdot\left[\begin{array}{c}
h_{1 i} \\
\vdots \\
h_{l-1 i} \\
0
\end{array}\right]=\sum_{k=1}^{l-1} h_{k i} p_{k}
$$

con $i=1, \ldots, l-1$ y $P \cdot\left[\begin{array}{cc}H & 0 \\ 0 & 0\end{array}\right] e_{n}=P \cdot 0=0$, por lo tanto $A \cdot P=P\left[\begin{array}{cc}H & 0 \\ 0 & 0\end{array}\right]$ y entonces $\left[\begin{array}{ll}P & 0 \\ 0 & 1\end{array}\right]^{-1}\left[\begin{array}{cc}A & p \\ -p^{t} & 0\end{array}\right]\left[\begin{array}{ll}P & 0 \\ 0 & 1\end{array}\right]=\left[\begin{array}{cc}p^{-1} A P & P^{-1} p \\ -p^{t} P^{-1} & 0\end{array}\right]$. Pero como $-p^{t} P=$ $[0, \ldots, 0,-\|p\|]$ y $P^{-1} p=\left[\begin{array}{c}0 \\ \vdots \\ 0 \\ \|p\|\end{array}\right]$ concluimos que:
$\left[\begin{array}{cc}P & 0 \\ 0 & 1\end{array}\right]^{-1}\left[\begin{array}{cc}A & p \\ -p^{t} & 0\end{array}\right]\left[\begin{array}{ll}P & 0 \\ 0 & 1\end{array}\right]=\left[\begin{array}{cc}H & 0 \\ 0 & 0 \\ 0 & \|p\| \\ -\|p\| & 0\end{array}\right]$

Así

$\operatorname{det}\left[\begin{array}{cc}A & p \\ -p^{t} & 0\end{array}\right]=(\operatorname{det} H) \cdot\|p\|^{2}=\|p\|^{2} \operatorname{det}\left(A / T_{p}\right), \operatorname{luego} \operatorname{det}\left(A / T_{p}\right)=\frac{1}{\|p\|^{2}} \operatorname{det}\left[\begin{array}{cc}A & p \\ -p^{t} & 0\end{array}\right]$.

Proposición 8 Sea $\mathcal{E}$ una economía de intercambio con una función $f$ de exceso de demanda agregada $C^{1}$, entonces

(i) $\partial f(p) \cdot p=0$

(ii) Si $p$ es un equilibrio $(f(p)=0)$, entonces $\partial f(p)$ mapea $\mathbb{R}^{l}$ en $T_{p}=\left\{q \in \mathbb{R}^{l} / p \cdot q=0\right\}$.

En este caso tenemos $\operatorname{det}\left(\partial f(p) / T_{p}\right)=\frac{1}{\|p\|^{2}} \operatorname{det}\left[\begin{array}{cc}\partial f(p) & p \\ -p^{t} & 0\end{array}\right]$.

En particular $p$ es un equilibrio regular si y sólo si $\partial f(p): T_{p} \longrightarrow T_{p}$ es invertible, lo cual es equivalente a que $\operatorname{det}\left[\begin{array}{cc}\partial f(p) & p \\ -p^{t} & 0\end{array}\right] \neq 0$. 


\section{Prueba}

(a) Como $f(\alpha p)=f(p)$ para $\alpha>0$, derivando con respecto a $\alpha$ esta ecuación tenemos que $\partial f(\alpha p) \cdot p=0$ y haciendo $\alpha=1$ obtenemos $\partial f(p) \cdot p=0$

(b) Para probar que $\partial f(p)\left(\mathbb{R}^{l}\right) \subseteq T_{p}$ considere $v=\left(v_{1}, \ldots, v_{l}\right) \in \mathbb{R}^{l}$ y sea $e_{1}, \ldots, e_{l}$ la base canónica de $\mathbb{R}^{l}$, entonces

$$
(\partial f(p) \cdot v) p=\left(\partial f(p)\left(\sum_{i=1}^{l} v_{i} e_{i}\right)\right) \cdot p=\sum_{i=1}^{l} v_{i}\left(\left(\partial f(p) e_{i}\right) p\right) .
$$

Por otro lado sea $G(p)=f(p) \cdot p=0$, entonces

$$
0=\frac{\partial G}{\partial p_{i}}=\frac{\partial f}{\partial p_{i}}(p) \cdot p+f(p) e_{i}=\frac{\partial f}{\partial p_{i}}(p) \cdot p
$$

Como $\left(\partial f(p) e_{i}\right) p=\frac{\partial f}{\partial p_{i}}(p) \cdot p$ para todo $i$, implica que $(\partial f(p) v) \cdot p=0$ para todo $v \in \mathbb{R}^{l}$, es decir $\partial f(p)(v) \in T_{p}$. Aplicando el Lema 7 a $A=\partial f(p)$ obtenemos

$$
\operatorname{det}\left(\partial f(p) / T_{p}\right)=\frac{1}{\|p\|^{2}} \operatorname{det}\left[\begin{array}{cc}
\partial f(p) & p \\
-p^{t} & 0
\end{array}\right] .
$$

El resto de la proposición sigue inmediatamente de lo anterior.

Definición 7 Sea $p$ un equilibrio de precios regular, definimos

$$
\text { índice }(p)=\operatorname{signo}\left(\operatorname{det}\left[\begin{array}{cc}
-\partial f(p) & p \\
-p^{t} & 0
\end{array}\right]\right) \text {. }
$$

Observe que

$$
\text { índice }(p)=(-1)^{l-1} \operatorname{signo}\left(\operatorname{det}\left[\begin{array}{cc}
\partial f(p) & p \\
-p^{t} & 0
\end{array}\right]\right)=\operatorname{signo}\left(\operatorname{det}\left(\partial(-f)(p) / T_{p}\right)\right)
$$

dado que

$$
\begin{aligned}
\operatorname{signo}\left(\operatorname{det}\left[\begin{array}{cc}
-\partial f(p) & p \\
-p^{t} & 0
\end{array}\right]\right) & =\operatorname{signo}\left((-1)^{l} \operatorname{det}\left[\begin{array}{cc}
\partial f(p) & -p \\
-p^{t} & 0
\end{array}\right]\right) \\
& =(-1)^{l}(-1) \operatorname{signo}\left(\operatorname{det}\left[\begin{array}{cc}
\partial f(p) & p \\
-p^{t} & 0
\end{array}\right]\right) \\
& =(-1)^{l-1} \operatorname{signo}\left(\operatorname{det}\left[\partial f(p) / T_{p}\right]\right) \\
& =\operatorname{signo}\left(\operatorname{det}\left(\partial(-f)(p) / T_{p}\right)\right) .
\end{aligned}
$$


Proposición 9 Sea $p$ un vector de equilibrio, para cada $q \in \mathbb{R}_{+}^{l} \backslash\{0\}$ tenemos que $p$ es un equilibrio regular si sólo si det $\left[\begin{array}{cc}\partial f(p) & q \\ q^{t} & 0\end{array}\right] \neq 0$. En particular el signo de este determinante no depende de $q$.

Prueba Sea $p$ un equilibrio regular y suponga que la matriz $\left[\begin{array}{cc}\partial f(p) & q \\ q^{t} & 0\end{array}\right]$ es singular, luego existe un vector $\left[\begin{array}{l}v \\ r\end{array}\right] \neq 0$, con $r \in \mathbb{R}$ y $v \in \mathbb{R}^{l}$ tal que $\partial f(p) v+r q=0$ y $q^{t} \cdot v=0$, multiplicando a la derecha por $p$ la primera ecuación se obtiene $p \cdot \partial f(p) v+r p \cdot q=0$, como $p \cdot \partial f(p)=0$ y $p \cdot q>0$ se debe tener $r=0$ y por tanto $v \neq 0$ y $\partial f(p) \cdot v=0$.

Los vectores $v$ y $p$ son linealmente independientes, ya que si $v=\alpha p$ y $q^{t} \cdot v=0$, tenemos $\alpha q^{t} p=0$ y siendo $q^{t} \cdot p>0$, implica que $\alpha=0$ y $v=0$, lo cual es una contradicción.

El núcleo de $\partial f(p)$ contiene a $v$ y $p$ y debe tener al menos dimensión 2 y por lo tanto $\operatorname{dim}($ rango $\partial(p))<l-1$ y $p$ no es regular, contradicción.

Para la otra implicación tome $q=p$ y entonces

$$
\operatorname{det}\left[\begin{array}{cc}
\partial f(p) & p \\
-p^{t} & 0
\end{array}\right]=(-1) \operatorname{det}\left[\begin{array}{cc}
\partial f(p) & p \\
p^{t} & 0
\end{array}\right] \neq 0
$$

luego $p$ es regular. La última parte de la proposición sigue de la continuidad del determinante.

Corolario 10 Sea $p$ un equilibrio regular, entonces el índice de $p$ está dado por el signo del determinante de la matriz obtenida de eliminar la $i$-ésima fila y columna de la matriz $-\partial f(p)$, con $1 \leq i \leq l$.

\section{Prueba}

$$
\begin{aligned}
\operatorname{indice}(p) & =\operatorname{signo}\left(\operatorname{det}\left[\begin{array}{cc}
-\partial f(p) & p \\
-p^{t} & 0
\end{array}\right]\right) \\
& =\operatorname{signo}\left((-1)^{l} \operatorname{det}\left[\begin{array}{cc}
\partial f(p) & p \\
p^{t} & 0
\end{array}\right]\right) \\
\left(e_{i} \text { vector de la base canónica de } \mathbb{R}^{l}\right) & =\operatorname{signo}\left((-1)^{l} \operatorname{det}\left[\begin{array}{cc}
\partial f(p) & e_{i} \\
e_{i}^{t} & 0
\end{array}\right]\right) \\
& =\operatorname{signo}\left(\operatorname{det}\left[\begin{array}{cc}
-\partial f(p) & e_{i} \\
-p_{i}^{t} & 0
\end{array}\right]\right) \\
& =\operatorname{signo}\left((-1) \operatorname{det}\left[\begin{array}{cc}
-\partial f(p) & e_{i} \\
e_{i} & 0
\end{array}\right]\right) \\
& =\operatorname{signo}((-1)(-1) \operatorname{det}[-\partial f(p)(i / i)])
\end{aligned}
$$

$(-\partial f(p)(i / i)$ es la matriz obtenida de $-\partial f(p)$ eliminando la fila y columna $i)$

$$
=\operatorname{signo}([-\partial f(p)(i / i)]) \text {. }
$$


Para la función de exceso de demanda agregada sabemos que $f(p)=f(p /\|p\|)$, por lo tanto el problema de buscar soluciones a la ecuación $f(p)=0$, se reduce a buscar soluciones de esta ecuación en $S_{++}^{l-1}=\left\{p \in \mathbb{R}_{++}^{l} /\|p\|=1\right\}$. Por esta razón de aquí en adelante supondremos que el dominio de $f$ es $S_{++}^{l-1}$. Matemáticamente la función $f: S_{++}^{l-1} \longrightarrow \mathbb{R}^{l}$ para precios normalizado puede verse como un campo vectorial tangente a $S_{++}^{l-1}$, es decir para cada $p \in S_{++}^{l-1}$ tenemos $f(p) \in T_{p}\left(S_{++}^{l-1}\right)=\left\{q \in \mathbb{R}^{l} / p \cdot q=0\right\}$, esto debido al hecho que para todo $p, f(p) \cdot p=0$.

Definición 8 Se denota por $T S_{++}^{l-1}$ al conjunto $\left\{(p, v) \in S_{++}^{l-1} \times \mathbb{R}^{l} / p \cdot v=0\right\} \quad$ y por $\left(T S_{++}^{l-1}\right)_{0}$ al conjunto $\left\{(p, v) \in T S_{++}^{l-1} / v=0\right\}$.

Definición 9 El gráfico de $f: S_{++}^{l} \longrightarrow \mathbb{R}^{l}$ es el conjunto

$$
\operatorname{Graf}(f)=\left\{(p, v) \in S_{++}^{l} \times \mathbb{R}^{l} / v=f(p)\right\}
$$

\section{Observaciones}

(a) $T S_{++}^{l-1}$ es una variedad $C^{\infty}$ de dimensión $2(l-1),\left(T S_{++}^{l-1}\right)_{0}$ es una subvariedad de $T S_{++}^{l-1}$ canónicamente difeomorfa a $S_{++}^{l-1}$ vía la función $g(v)=(v, 0)$ y por lo tanto $T_{(p, 0)}\left(T S_{++}^{l-1}\right)_{0}=T_{p} \times\{0\}$.

(b) $\operatorname{Graf}(f)$ es una subvariedad $C^{1}$ de $T S_{++}^{l-1}$, canónicamente difeomorfa a $S_{++}^{l-1}$ vía $h(p)=$ $(p, f(p))$. Para $p \in S_{++}^{l-1}$ tenemos que $T_{h(p)}(\operatorname{Graf}(f))=\operatorname{Graf}(\partial f(p))=\{(v, \partial f(p) v) / v \in$ $\left.T_{p}\right\}$ y $\partial h(p)(v)=(v, \partial f(p)(v))$.

Todas las pruebas de estos resultados pueden ser encontradas en Guillermin-Pollack, 1974 .

$h$ vista como función de $S_{++}^{l-1} \longrightarrow T S_{++}^{l-1}$ es tal que $h^{-1}\left(\left(T S_{++}^{l-1}\right)_{0}\right)$ es exactamente el conjunto de los vectores de precios de equilibrio en $S_{++}^{l-1}$. Denotemos tal conjunto por E.

Proposición 11 El conjunto $E$ de precios de equilibrio de una economía $\mathcal{E}$ en $S_{++}^{l-1}$ es compacto.

Prueba Es suficiente probar que $E$ es cerrado y acotado. Como $E \subseteq S_{++}^{l-1}$ es acotado, sea $p_{n} \in E$ secuencia tal que $p_{n} \longrightarrow p,\|p\|=1$, entonces $p \neq 0$ y por la parte (iv) de la proposición 5 , dado que $\left\|f\left(p_{n}\right)\right\|=0$, para todo $n$, debemos tener $p>0$, luego $p \in S_{++}^{l-1}$. Por continuidad de $f, f(p)=\lim _{n \rightarrow \infty} f\left(p_{n}\right)=0$, consecuentemente $p \in E$ y $E$ es cerrado.

Si en la proposición anterior reemplazamos $S_{++}^{l-1}$ por $\mathbb{R}_{++}^{l}$, el resultado es falso ya que si $p$ es un vector de equilibrio $f(\alpha p)=0$ para todo $\alpha$. En particular $\alpha p \longrightarrow 0$ si $\alpha \longrightarrow 0$, por lo tanto el conjunto de precios de equilibrio en $\mathbb{R}_{++}^{l}$ no es cerrado si no es vacío, por lo tanto no sería compacto.

Proposición 12 Si $p$ es un vector de precios de equilibrio regular, entonces $p$ es un punto aislado de $E$.

Prueba Sea $p$ tal que $f(p)=0$ y tal que el rango de $\partial f(p)$ es igual a $l-1$. Si se considera $h: S_{++}^{l-1} \longrightarrow T S_{++}^{l-1} \operatorname{con} h(q)=(q, f(q))$, tenemos $h(p)=(p, 0) \in\left(T S_{++}^{l-1}\right)_{0}$. La codimensión 
de $\left(T S_{++}^{l-1}\right)_{0}$ en $T S_{++}^{l-1}$ es $l-1$, luego existe un abierto $V$ en $T S_{++}^{l-1}$ y $g: V \longrightarrow \mathbb{R}^{l-1}$ tal que $(p, 0) \in V, g$ es $C^{1}$, para todo $v \in V, \partial g(v)$ es sobreyectiva y $g^{-1}(0)=V \cap\left(T S_{++}^{l-1}\right)_{0}$.

Si $r=g \circ h: h^{-1}(V) \longrightarrow V \longrightarrow \mathbb{R}^{l-1}$ afirmamos que $\partial r(p): T_{p} \longrightarrow \mathbb{R}^{l-1}$ es biyectiva. Es suficiente probar que $\partial r(p)$ es sobreyectiva ya que $\operatorname{dim} T_{p}=\operatorname{dim} \mathbb{R}^{l-1}=l-1$.

Por la regla de la cadena $\partial r(p)=\partial g(h(p)) \circ \partial h(p)$, es decir el siguiente diagrama conmuta.

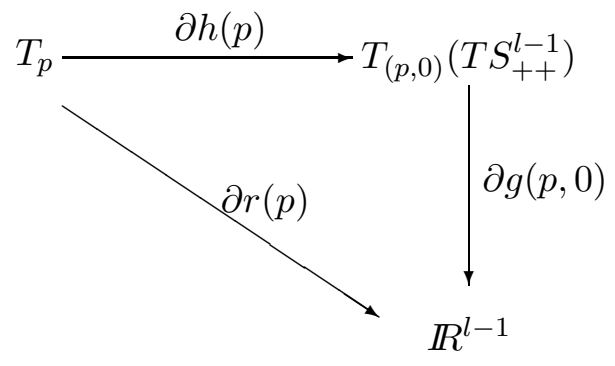

Calculemos $T_{(p, 0)}\left(T S_{++}^{l-1}\right)$; considere $s: S_{++}^{l-1} \times \mathbb{R}^{l} \longrightarrow \mathbb{R}$ tal que $s(p, v)=p \cdot v$, es claro que $\partial s(p, 0): T_{p} \times \mathbb{R}^{l} \longrightarrow \mathbb{R}$, es dado por $\partial s(p, 0)\left(v, v^{\prime}\right)=v^{\prime} \cdot p$. Como $\left(T S_{++}^{l-1}\right) \cap V=s^{-1}(0)$, entonces:

$T_{(p, 0)}\left(T S_{++}^{l-1}\right)=T_{(p, 0)}\left(T S_{++}^{l-1} \cap V\right)=\operatorname{ker}(\partial s(p, 0))=\left\{\left(v, v^{\prime}\right) \in T_{p} \times \mathbb{R}^{l} / v^{\prime} \cdot p=0\right\}=T_{p} \times T_{p}$.

Por otro lado $T_{(p, 0)}\left(T S_{++}^{l-1}\right)_{0}=T_{p} \times\{0\}$. Como $p$ es regular, entonces $\partial f(p)\left(T_{p}\right)=T_{p}$, y si $\left(v, v^{\prime}\right) \in T_{p} \times T_{p}$ existe $u \in T_{p}$ con $\partial f(p)(u)=v^{\prime}$, luego

$$
\left(v, v^{\prime}\right)=(v-u, 0)+(u, \partial f(p)(u)) \in T_{p} \times\{0\}+\operatorname{graf}(\partial f(p)) .
$$

Por lo tanto $T_{p} \times\{0\}+\operatorname{graf}(\partial f(p))=T_{p} \times T_{p}$. Como $T_{(p, 0)}\left(T S_{++}^{l-1}\right)_{0}=\operatorname{ker} \partial g(p, 0)$ y $\operatorname{graf}(\partial f(p))=\operatorname{imagen}(\partial h(p))$, entonces tenemos

$$
\partial g(p, 0)(\text { imagen }(\partial h(p)))=\partial g(p, 0)\left(T_{p} \times T_{p}\right)=\mathbb{R}^{l-1}
$$

y $\partial r(p)$ es sobreyectiva. Por el teorema de la función inversa, existe un abierto $U$ de $S_{++}^{l}$ tal que $p \in U$ y $r / U: U \longrightarrow \mathbb{R}^{l-1}$ es uno a uno, entonces

$$
\{p\}=(r / U)^{-1}(0)=r^{-1}(0) \cap U=h^{-1}\left(\left(T S_{++}^{l-1}\right)_{0}\right) \cap U=E \cap U,
$$

es decir $p$ es un punto aislado de $E$.

Corolario 13 El conjunto de precios de equilibrio regular de una economía $\mathcal{E}$ es discreto.

Proposición 14 Si la economía $\mathcal{E}$ es regular, el conjunto de precios de equilibrio es finito. Prueba Por el corolario 13 tal conjunto es discreto y por la proposición 11 es compacto, luego debe ser finito.

Lema 15 Sea $p_{m} \longrightarrow p$ una secuencia convergente de puntos en $S_{++}^{l-1}, p \neq 0$ en la frontera topológica de $S_{++}^{l-1}$ y $f\left(p_{m}\right) /\left\|f\left(p_{m}\right)\right\| \longrightarrow z$, entonces $z \geq 0$, más aún si $z_{j}>0$ entonces $p_{j}=0$. 
Prueba Sabemos de parte (iv) de la proposición 2 que $\left\|f\left(p_{m}\right)\right\| \longrightarrow \infty$, cuando $m \longrightarrow \infty$. Por otro lado, para todo $q \in \mathbb{R}_{++}^{l}, f(q)>-\sum_{t=1}^{n} w_{t}$, entonces para todo $j$ se tiene que $f^{j}\left(p_{m}\right)>-\sum_{t=1}^{n} w_{t}^{j}$, luego $f^{j}\left(p_{m}\right) /\left\|f\left(p_{m}\right)\right\|>-\sum_{t=1}^{n} w_{t}^{j} /\left\|f\left(p_{m}\right)\right\|$, pasando al límite $z_{j} \geq 0$. Como $z \neq 0$ se tiene $z \geq 0$. Suponga que $z_{j}>0$, como $f\left(p_{m}\right) \cdot p_{m}=0$, entonces $\left(f\left(p_{m}\right) /\left\|f\left(p_{m}\right)\right\|\right) \cdot p_{m}=0$, pasando al límite $z \cdot p=0$. Dado que para todo $i, p_{i} \geq 0$ y $z_{i} \geq 0$, entonces $0 \leq p_{j}=-\sum_{k \neq j} \frac{p_{k} z_{k}}{z_{j}} \leq 0$, luego $p_{j}=0$.

Sea $e=(1, \ldots, 1)$, defina $h: S_{++}^{l-1} \longrightarrow \mathbb{R}^{l}$ tal que $h(p)=(e-(e \cdot p) \cdot p) / \sqrt{n}, h$ es un campo vectorial tangente en $S_{++}^{l-1}$, dado que $h(p) \cdot p=\frac{(e \cdot p)-(e \cdot p)}{\sqrt{n}}=0$ y $h(p)$ claramente apunta en la dirección a $\frac{e}{\sqrt{n}} \in S_{++}^{l-1}$.

Denote por $S_{\epsilon}$ el conjunto $\left\{p \in S_{++}^{l-1} /\right.$ para todo $\left.j, \quad p_{j} \geq \epsilon\right\}$.

Proposición 16 Para $\epsilon>0$ pequeño, las funciones $f$ y $h$ son homotópicas en el complemento de $S_{\epsilon}$ en $S_{++}^{l-1}$, más aún $\alpha f(p)+(1-\alpha) h(p) \neq 0$ para todo $p \in S_{++}^{l-1}$ fuera de $S_{\epsilon} \mathrm{y}$ $0 \leq \alpha \leq 1$.

Prueba Es suficiente probar que $\alpha f(p)+(1-\alpha) h(p) \neq 0$ en el complemento de $S_{\epsilon}$ en $S_{++}^{l-1}$, para $\epsilon$ pequeño, ya que $H(p, \alpha)=\frac{\alpha f(p)+(1-\alpha) h(p)}{\|\alpha f(p)+(1-\alpha) h(p)\|}$ define una homotopía entre $f \mathrm{y} h$.

Suponga por contradicción, que para todo $n$ entero positivo existe $p_{n} \in S_{++}^{l-1}$ tal que $p_{n} \notin S_{1 / n}$ y $\alpha f\left(p_{n}\right)+(1-\alpha) h\left(p_{n}\right)=0$. Como $\left\|p_{n}\right\|=1$, podemos suponer que $p_{n} \longrightarrow$ $p \in \overline{S_{++}^{l-1}}$ y como $p_{n} \notin S_{1 / n}$, tenemos que $0<\min \left\{p_{n}^{j} / 1 \leq j \leq l\right\}<1 / n$, luego $p_{j_{0}}=$ $\operatorname{mín}\left\{p_{j} / 1 \leq j \leq l\right\}=\lim _{n \rightarrow \infty} \operatorname{mín}\left\{p_{n}^{j} / 1 \leq j \leq l\right\}=0$ y entonces $p$ pertenece a la frontera topológica de $S_{++}^{l-1}$ y por el lema $15, f\left(p_{n}\right) /\left\|f\left(p_{n}\right)\right\| \longrightarrow z \geq 0$. Sea $j$ tal que $z_{j}>0$, entonces $f^{j}\left(p_{n}\right) /\left\|f\left(p_{n}\right)\right\| \longrightarrow z_{j}>0$ y existe $n_{0}$ entero positivo tal que para $n \geq n_{0}$ $f^{j}\left(p_{n}\right) /\left\|f\left(p_{n}\right)\right\|>z_{j} / 2>0$, luego $f^{j}\left(p_{n}\right)>0$. Como $z_{j}>0$ por lema $15, p_{j}=0$ y dado que $h^{j}\left(p_{n}\right)=\frac{1-\left(\sum_{i} p_{n}^{i}\right) p_{n}^{j}}{\sqrt{n}}, h^{j}\left(p_{n}\right) \longrightarrow \frac{1}{\sqrt{n}}>0$, existe $n_{1}$ entero positivo tal que si $n \geq n_{1}$, $h^{j}\left(p_{n}\right)>0$. Así tenemos que para $n \geq \operatorname{máx}\left\{n_{0}, n_{1}\right\}, \alpha f^{j}\left(p_{n}\right)+(1-\alpha) h^{j}\left(p_{n}\right)>0$, para todo $\alpha \in[0,1]$, pero esto contradice la ecuación $\alpha f\left(p_{n}\right)+(1-\alpha) h\left(p_{n}\right)=0$.

Sea $C_{i}^{\delta, \epsilon}=\left\{\left(x_{1}, \ldots, x_{l}\right) / \sum_{k \neq i}\left(x_{k}-\epsilon-\delta\right)^{2}>\delta^{2}\right.$ y para todo $\left.k \neq i, \epsilon \leq x_{k}<\epsilon+\delta\right\}$, para $i=1, \ldots, l, l \geq 3, \delta, \epsilon>0 \mathrm{y} \epsilon+\delta<1 / 4$.

Si $\mathbb{R}_{+}^{l}=\left\{p \in \mathbb{R}^{l} / p_{i} \geq 0\right.$ para todo $\left.i\right\}$, denote por $A_{i}^{\delta, \epsilon}$ el complemento de $C_{i}^{\delta, \epsilon}$ en $\mathbb{R}_{+}^{l}$. $A_{i}^{\delta, \epsilon}$ es una variedad $C^{1}$ con frontera, por lo tanto $A_{i}^{\delta, \epsilon} \cap S_{++}^{l-1}$ es una variedad con frontera $C^{1}$. Así $B^{\delta, \epsilon}=\bigcap_{i=1}^{l}\left(A_{i}^{\delta, \epsilon} \cap S_{++}^{l-1}\right)$ es una variedad con frontera $C^{1}$ difeomorfa al disco $\left\{x \in \mathbb{R}^{l-1} /\|x\| \leq 1\right\}$ en $\mathbb{R}^{l-1}$.

Para $n=3, B^{\delta, \epsilon}$ es la región más pequeña de $S_{++}^{2}$ que aparece en la figura. 


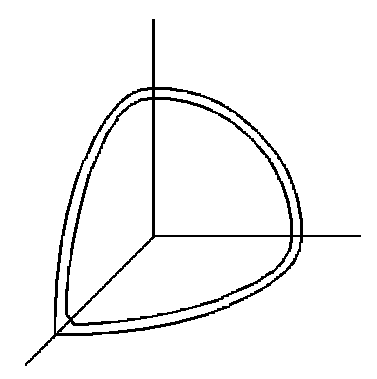

Si $l \leq 2$, sea $B^{\delta, \epsilon}=S_{\delta \cdot \epsilon}$.

Teorema 17 (Teorema del índice) Si $E$ es el conjunto de precios de equilibrio en una economía regular $\mathcal{E}$, entonces $\sum_{p \in E}$ índice $(p)=1$.

Prueba Sea $\epsilon^{\prime}>0$ suficientemente pequeño tal que $f$ y $h$ sean homotópicas en el complemento de $S_{\epsilon^{\prime}}$ en $S_{++}^{l-1}$. Escoja $\epsilon$ y $\delta$ pequeños tales que la frontera de $B^{\delta, \epsilon}$ está contenida en el complemento de $S_{\epsilon^{\prime}}$ en $S_{++}^{l-1}$ y $E$ contenido en el interior de $B^{\delta, \epsilon}$. Tenemos entonces que $f$ y $h$ son homotópicas en la frontera de $B^{\delta, \epsilon}$ y $h$ apunta al interior de $B^{\delta, \epsilon}$. Por lo tanto, módulo homotopía - $f$ apunta al exterior de $B^{\delta, \epsilon}$ en la frontera de $B^{\delta, \epsilon}$. Aplicando el teorema de Poincaré-Hopf (ver apéndice) a $-f$ y $B^{\delta, \epsilon}$ se tiene que

$$
\sum_{p \in E} \operatorname{signo}(\operatorname{det}(-\partial f(p)))=\chi\left(B^{\delta, \epsilon}\right)
$$

donde $\chi\left(B^{\delta, \epsilon}\right)$ denota la característica de Euler de $B^{\delta, \epsilon}$. Dado que $B^{\delta, \epsilon}$ es difeomorfa a la bola unitaria en $\mathbb{R}^{l-1}$ se tiene que $\chi\left(B^{\delta, \epsilon}\right)=1$ (la característica de Euler de bola unitaria es 1) y por lo tanto $\sum_{p \in E}$ índice $(p)=1$.

Corolario 18 En toda economía regular, el conjunto $E$ de los precios de equilibrio tiene un número impar de elementos. En particular $E \neq \emptyset$.

Prueba Como índice $(p)$ es 1 ó -1, y $\sum_{p \in E}$ índice $(p)=1$, entonces el número de veces que índice $(p)=1$ es uno más que el número de veces que índice $(p)=-1$, luego $|E|$ debe ser impar.

Corolario $19 E$ tiene un único elemento si y sólo si para cada $p \in E$, tenemos que

$$
\operatorname{signo}\left(\operatorname{det}\left[\begin{array}{cc}
\partial f(p) & p \\
-p^{t} & 0
\end{array}\right]\right)=(-1)^{l-1} \text {. }
$$

Prueba $(\Longrightarrow)$ Sea $p \in E$, por el teorema del índice $\sum_{q \in E}(-1)^{l-1} \operatorname{signo}\left(\operatorname{det}\left(\partial f(q) / T_{p}\right)\right)=$ 1 , pero como $E=\{p\}$, entonces $(-1)^{l-1} \operatorname{signo}\left(\operatorname{det}\left(\partial f(q) / T_{p}\right)\right)=1$. Así signo $\left(\operatorname{det}\left(\partial f(q) / T_{p}\right)\right)=$ $(-1)^{l-1}$, para todo $p \in E$.

Recíprocamente, si signo $\left(\operatorname{det}\left(\partial f(q) / T_{p}\right)\right)=(-1)^{l-1}$ para todo $p \in E$, por la fórmula de índice obtenemos $\sum_{p \in E} 1=1$, es decir $|E|=1$.

Nota Si para cada $p \in E$ en una economía regular, $\partial f(p)$ es negativa quasidefinida en $T_{p}$, esto es $v^{t} \cdot \partial f(p) v<0$ para todo $v \neq 0$ en $T_{p}$, entonces se satisface la condición del corolario 19 (toda matriz $m \times m$ negativa quasidefinida, su determinante tiene signo $(-1)^{m}$, ver McKenzie, 1960) y por lo tanto el equilibrio es único. 


\section{Apéndice}

\section{Definición}

(a) Una orientación para un espacio vectorial real $V$ de dimensión finita $n$, es una clase de equivalencia en el conjunto de bases ordenadas de $V$, donde la relación de equivalencia es dada por: $\left(b_{1}^{\prime}, \ldots, b_{n}^{\prime}\right)$ determina la misma orientación que la base $\left(b_{1}, \ldots, b_{n}\right)$, si $b_{i}^{\prime}=\sum_{j} a_{i j} b_{j}$ con $\operatorname{det}\left(a_{i j}\right)>0$. La orientación opuesta se $\operatorname{determina~si~} \operatorname{det}\left(a_{i j}\right)<0$.

Todo espacio vectorial de dimensión finita tiene exactamente dos orientaciones. La orientación canónica en $\mathbb{R}^{n}$ corresponde a la base $(1,0, \ldots, 0), \ldots,(0, \ldots, 0,1)$.

(b) Una variedad diferencial $C^{r}$ orientada consiste de una variedad $M$ junto con una elección de orientación para cada $T_{x}(M)$ (espacio tangente de $M$ en $x$ ), tal que para cada $x \in M$ existe un vecindario abierto $U$ de $x$ en $M$ y un difeomorfismo $C^{r}, h$ que mapea $U$ sobreyectiva en un abierto de $\mathbb{R}^{n}$ ó $H^{n}=\left\{\left(x_{1}, \ldots, x_{n}\right) / x_{n} \geq 0\right\}$, de tal manera que $\partial h(x)$ manda la orientación de $T_{x}(M)$ en la orientación canónica de $\mathbb{R}^{n}$. Si $M$ es convexo $M$ tiene exactamente dos orientaciones.

(c) Sean $M, N$ variedades $C^{r} n$-dimensionales orientadas sin frontera y sea $f: M \longrightarrow N$ una función $C^{r}$. Si $M$ es compacto y $N$ conexo, entonces el grado de $f$ se define de la siguiente manera $\operatorname{grado}(f)=\sum_{x \in f^{-1}(y)} \operatorname{signo}(\partial f(x))$, donde $y \in N$ es tal que $x \in f^{-1}(y)$ implica que $\partial f(x): T_{x}(M) \longrightarrow T_{y}(N)$ es un isomorfismo lineal ( $y$ siempre existe por el teorema de Sard, ver Milnor (1965)) y signo $(\partial f(x))$ se define como +1 si $\partial f(x)$ preserva orientación y como -1 si la invierte. El grado $(f)$ no depende de $y$.

Definición Sea $N \subseteq \mathbb{R}^{m}$ una variedad $n$-dimensional $C^{1}$ con frontera. Un campo vectorial tangencial $C^{r}$ en $N$ es una función $C^{r}, f: N \longrightarrow \mathbb{R}^{m}$ tal que $f(x) \in T_{x}(N)$ para todo $x \in N$.

Sea $U \subseteq \mathbb{R}^{s}$ un conjunto abierto y $v: U \longrightarrow \mathbb{R}^{s}$ un campo vectorial tangencial $C^{r}$ en $U$ con un cero $z(f(z)=0)$ aislado en $U$. La función $\bar{v}(x)=v(x) /\|v(x)\|$ mapea una pequeña esfera con centro $z$ en la esfera unitaria de $\mathbb{R}^{s}$, ambas esferas con la orientación canónica. El grado de esta función es llamada el índice $i(z)$ de $v$ en $z$.

Suponga que $X$ es una $n$-variedad $C^{1}$ orientada y $v$ un campo vectorial tangencial en $X$. Suponga que $x$ es un cero aislado de $v$ en $X$ y sea $\phi: U \longrightarrow X$ una parametrización alrededor de $x$ con $\phi(0)=x$. Para cada $u \in U$, la derivada $\partial \phi(n): \mathbb{R}^{n} \longrightarrow \mathbb{R}^{n}$ es un isomorfismo lineal. Denote $\phi^{*} v$ el campo vectorial en $U$ tal que $\phi^{*} v(u)=\left(\partial \phi_{u}\right)^{-1}(v(\phi(u)))$.

Como $v$ tiene un cero aislado en $x$, entonces $\phi^{*} v$ tiene un cero aislado en 0. Definimos el índice $i(x)$ de $\vec{v}$ en $x$ como el índice de $\phi^{*} v$ en 0 . Esta definición es independiente de la parametrización $\phi$ (ver Milnor (1965)).

Proposición A Si $x$ es un cero de un campo vectorial $v$ tangencial $C^{r}$, en una $n$-variedad diferencial $C^{1}, N$ entonces $\partial f(x)$ mapea $T_{x}(N)$ en $T_{x}(N)$ y si además $\partial f(x)$ es un isomorfismo lineal, tenemos que $i(z)=\operatorname{signo}(\operatorname{det}(\partial f(x)))$.

Teorema B (Teorema de Poincaré-Hopf) Sea $N$ una $n$ variedad compacta $C^{1}$, con frontera, orientada y $v$ un campo vectorial tangencial $C^{1}$ en $N$ tal que:

(i) $v$ apunta al exterior de $N$ en cada punto de la frontera de $N$ 
(ii) $v$ tiene un número finito de ceros,

entonces la suma de los índices de $v$ en cada cero es igual a la característica de Euler de $N$.

La versión 2-dimensional de este teorema fue probada por Poincaré en 1885. El teorema general fue probado por Hopf en 1926.

Si $N=B^{n}=\left\{x \in \mathbb{R}^{n} /\|x\| \leq 1\right\}$ el teorema sigue siendo válido si (i) se remplaza simplemante por el hecho de que $v / \partial B^{n}$ sea homotópica a una función $C^{1}$ que apunte al exterior de $B^{n}$. La característica de Euler de $B^{n}$ es 1 .

Para las pruebas de todos estos resultados y más información ver por ejemplo Milnor (1965) y Guillemin-Pollack (1974).

\section{Referencias}

[1] Acuña, Osvaldo y Ulate, F. 1991, Desarrollo, proposiciones y pruebas matemáticas sobre la teoría de demanda sin utilidad, Revista Ciencias Económicas Vol XI, No $1-2$.

[2] Acuña, Osvaldo y Ulate, F. 1992, Equilibrio de precios y óptimo de Pareto en Intercambio, Ciencias Matemáticas 3(1), pp. 77-90.

[3] Debreu, G. (1970) Economies with a finite set of equilibria, Econometrica 38(3), pp. 387-392.

[4] Dierker, E. (1972) Two remarks on the number of equilibria of an economy, Econometrica 40(5), pp. 951-953.

[5] Guillemin, V. and Pollack, A. (1974) Differential Topology. Englewood Cliffs, NJ, Prentice-Hall.

[6] Hopf, H. (1926) Vertorfelder in n-dimensionalen Mannigtalf y Keiten, Math Annalen, 96, pp. 225-250.

[7] Mas-Collel, A. (1985) The theory of general Economic Equilibrium. A differentiable approach. Cambridge University Press, Cambridge.

[8] McKenzie, L. (1960) Matrices with dominant diagonals and economic theory. In: Mathematical Methods in Social Sciences, 1959, K. Arrow, S. Karlin and P. Suppes (eds.), pp. 47-62. Stanford CA, Stanford University Press.

[9] Milnor, J. (1965) Topology from the Differentiable Viewpoint. Cherlottesville. University Press of Virginia.

[10] Walras, L. (1954) Elements of Pure Economics (1926). Trad. por W. Jaffí, London, Allen-Unwin. 